\title{
Light quark spectrum with improved gauge and fermion actions*
}

\author{
C. Bernard, ${ }^{\mathrm{a}}$ T. DeGrand,${ }^{\mathrm{b}}$ C. DeTar, ${ }^{\mathrm{c}}$ Steven Gottlieb,${ }^{\mathrm{d}}$ Urs M. Heller, ${ }^{\mathrm{e}}$ J. Hetrick, ${ }^{\mathrm{a}}$ C. McNeile, ${ }^{\mathrm{c}}$ \\ K. Rummukainen, ${ }^{\mathrm{f}}$ R. Sugar, ${ }^{\mathrm{g}}$ D. Toussaint, ${ }^{\mathrm{h}}$ and M. Wingate, ${ }^{\mathrm{b}}$ \\ ${ }^{a}$ Department of Physics, Washington University, St. Louis, MO 63130, USA \\ bPhysics Department, University of Colorado, Boulder, CO 80309, USA

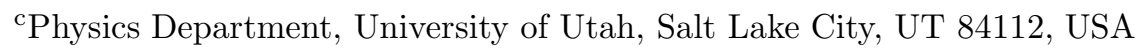 \\ d Department of Physics, Indiana University, Bloomington, IN 47405, USA \\ eSCRI, The Florida State University, Tallahassee, FL 32306-4130, USA \\ ${ }^{\mathrm{f}}$ Department of Physics, University of Bielefeld, D-33501 Bielefeld, Germany \\ gDepartment of Physics, University of California, Santa Barbara, CA 93106, USA \\ ${ }^{\mathrm{h}}$ Department of Physics, University of Arizona, Tucson, AZ 85721, USA
}

We report on a study of the light quark spectrum using an improved gauge action and both Kogut-Susskind and Naik quark actions. We have studied six different lattice spacings, corresponding to plaquette couplings ranging from 6.8 to 7.9 , with five to six quark masses per coupling. We compare the two quark actions in terms of the spectrum and restoration of flavor symmetry. We also compare these results with those from the conventional action.

For the past few years, it has been quite popular to add additional terms to the Wilson gauge action, or the Wilson or Kogut-Susskind quark actions in an attempt to reduce finite lattice spacing artifacts [1]. There has been considerable success in improving the Wilson quark action, which has errors of order $a$, by introducing the SW clover term [2] to produce an action with errors of order $a^{2}$. Here we report on what may be the more challenging problem of improving the Kogut-Susskind quark action, which only has errors of order $a^{2}$ to start with. We have extended the study we reported on last year to three weaker couplings [3].

For the gauge fields, we use a tadpole improved three-term action that includes the plaquette, $1 \times$ 2 rectangle and a six link term that corresponds to a path with steps $+x,+y,+z,-x,-y,-z$. The couplings for each term are denoted $\beta_{p l}, \beta_{r t}$ and $\beta_{p g}$.

\footnotetext{
*presented by S. Gottlieb
}

The Naik quark action [4] extends the staggered quark action to include a three-link derivative term in addition to the usual nearest neighbor derivative. If the coefficients of the one and three link terms are denoted $c_{1}$ and $c_{3}$, the KogutSusskind action is $c_{1}=1$ and $c_{3}=0$, while the tree-level tadpole-improved Naik action is $c_{1}=9 /\left(8 u_{0}\right)$ and $c_{3}=-1 /\left(24 u_{0}^{3}\right)$. Here $u_{0}$ is the tadpole improvement factor that we take to be the fourth root of the plaquette. The Naik action improves the free quark dispersion relation to remove order $a^{2}$ errors. Luo [5] has identified 15 independent dimension six lattice operators that might be added to Kogut-Susskind action to form an order $a^{2}$ improved action for the interacting theory. Thus, going beyond tree level improvement may require terms such as "fat link" interactions [6] and next-nearest neighbor interactions.

We have three new ensembles of $16^{3} \times 32$ lattices with $\beta_{p l}=7.6,7.75$ and 7.9. For 7.6, we have 100 lattices which were provided by the SCRI 


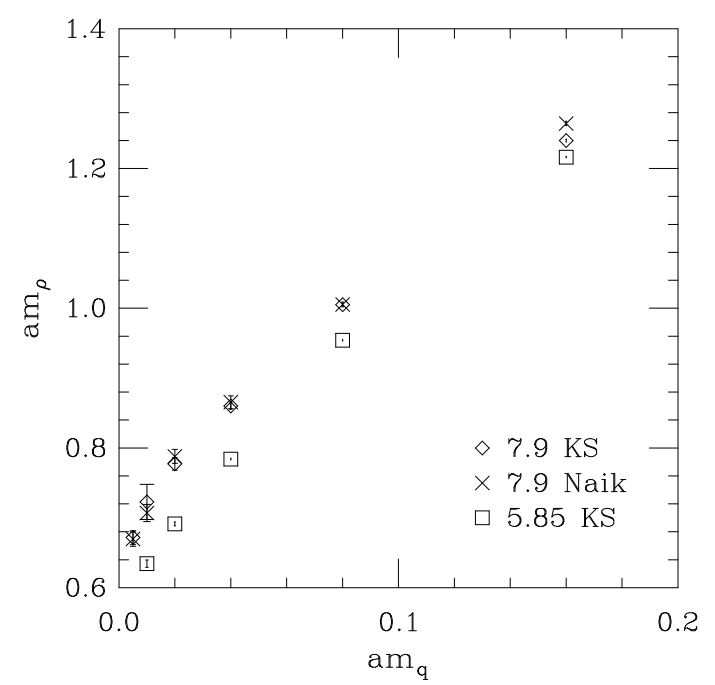

Figure 1. Rho mass vs. quark mass for standard and improved actions.

group [7]. For the other couplings we have 200 lattices. Our previous stronger coupling work used couplings $6.8,7.1$ and 7.4 , again with 200 lattice at each coupling. We used the same volume as above, except for 7.1 , where we used $14^{4} \times 28$. We found that 7.4 was the strongest coupling that gave a reasonable Edinburgh plot [3], so we have moved on to weaker couplings. Results at 7.6 were not ready in time for the conference, but are included in some of the graphs for completeness.

For each ensemble we have computed hadron propagators using five or six quark masses differing by a factor of two. At the strongest couplings the hadrons are quite heavy in lattice units. For instance at 7.1, $a m_{N}$ is between 2 and 3.4, while $a m_{\rho}$ is between 1.3 and 2.2. By 7.4, the $\rho$ mass extrapolated to $a m_{q}=0$ is about 1.2 and the nucleon is 1.7. This $\rho$ mass is comparable to $6 / g^{2}=5.54$ for the standard Wilson gauge action and staggered quarks. In Fig. 1, we compare our weakest coupling 7.9 , with the standard action $6 / g^{2}=5.85$. We see that 5.85 has a somewhat lighter rho, and hence we infer a smaller lattice spacing. Thus, our improved action results correspond to a slightly smaller range than $5.54-5.85$ in terms of the standard action. In terms of the

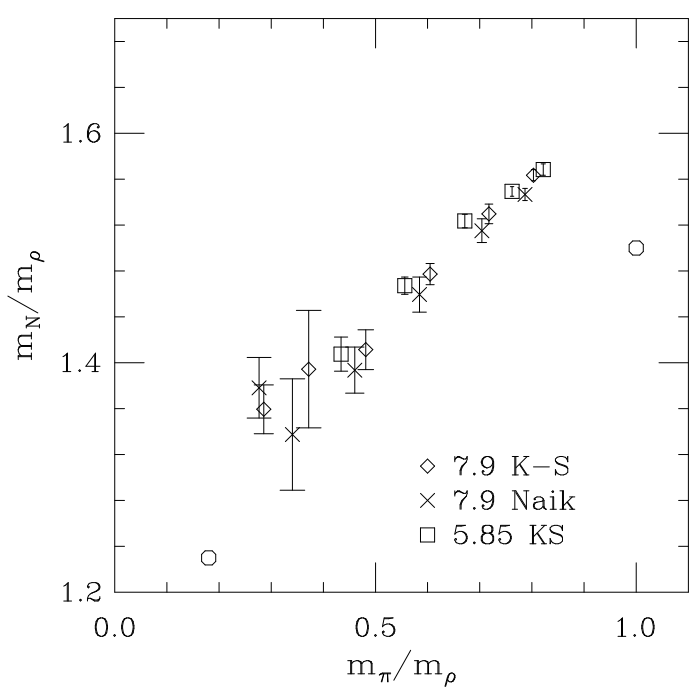

Figure 2. Edinburgh plot comparing the standard action with $6 / g^{2}=5.85$ and the improved actions with $\beta_{p l}=7.9$.

lattice spacing, this range is roughly $0.3-0.15 \mathrm{fm}$.

We compare Edinburgh plots for $6 / g^{2}=5.85$ with $\beta_{p l}=7.9$ in Fig. 2. Despite the fact that 5.85 has a smaller lattice spacing (as determined by the $\rho$ mass), the results for the improved gluonic action give a nucleon to rho mass ratio that may be slightly lower. This improvement is actually clearer at stronger couplings as we shall see later.

We next turn to the issue of flavor symmetry restoration. We have found that adding "fat link" terms to the fermion action can result in a substantial improvement in flavor symmetry [6]. In Fig. 3, we display $\delta_{\pi}=\left(m_{\pi_{2}}^{2}-m_{\pi}^{2}\right) / M_{\rho}^{2}$ vs. quark mass for our standard staggered spectrum with $6 / g^{2}=5.54,5.7,5.85$ and 6.15 , and for the improved gluonic action with either KogutSusskind or Naik quarks. Except at the largest quark mass, $\beta_{p l}=7.4$ clearly has smaller values of $\delta_{\pi}$ than the comparable standard coupling 5.54. For $\beta_{p l}=7.9, \delta_{\pi}$ is smaller than the standard action 5.85 results, which are the crosses between the octagons and squares for 7.75 and 7.9. Thus, we see improvement in flavor symmetry restoration, although it seems to be coming more from the improvement in the gluonic action than the 


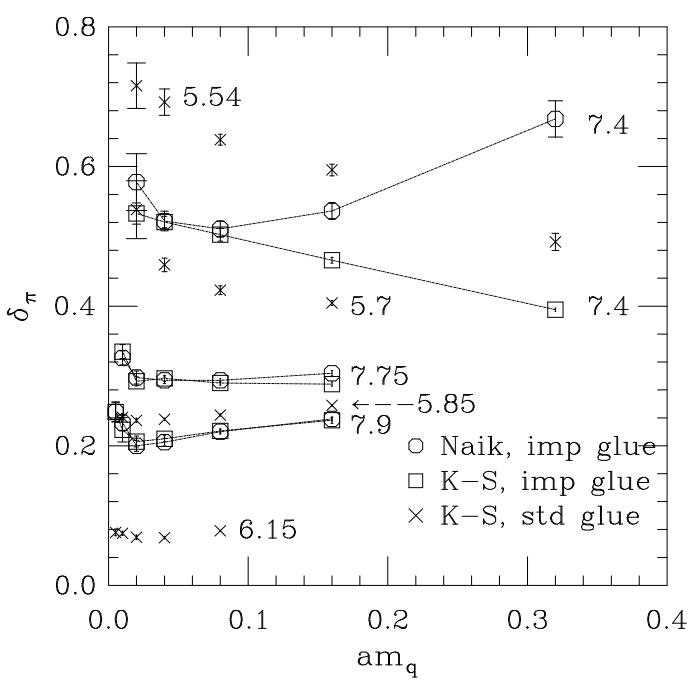

Figure 3. Flavor symmetry breaking variable $\delta_{\pi}$ vs. quark mass for various actions and couplings.

quark action.

For the Wilson action quenched hadron spectrum, determining the correct form of the chiral extrapolation has been a crucial issue. This problem is also present for the current calculations, but there is insufficient space for a full discussion, and our study of this issue has not yet been completed. At the conference we showed graphs of the nucleon to rho mass ratio extrapolated to physical quark mass vs. am $\rho$. Such graphs yielded scant encouragement as to the value of the current attempt at improvement. In view of the difficulty in controlling the chiral extrapolation, we display here a chiral interpolation to $m_{\pi} / m_{\rho}=0.5$. The crosses represent the standard gluonic action. A curve with the expected quadratic lattice spacing dependence is drawn. From left to right, the couplings are $6.15,5.85,5.7,5.54$. The results with improved glue are all below the standard curve. For the two stronger couplings, $\beta_{p l}=7.6$ and 7.4, there is a noticeable difference between the Naik and Kogut-Susskind actions. However, for the two weaker couplings, 7.75 ad 7.9 , they are indistinguishable on this plot. It is possible that by adding terms such as suggested by Luo, one might be able to further improve the quark action

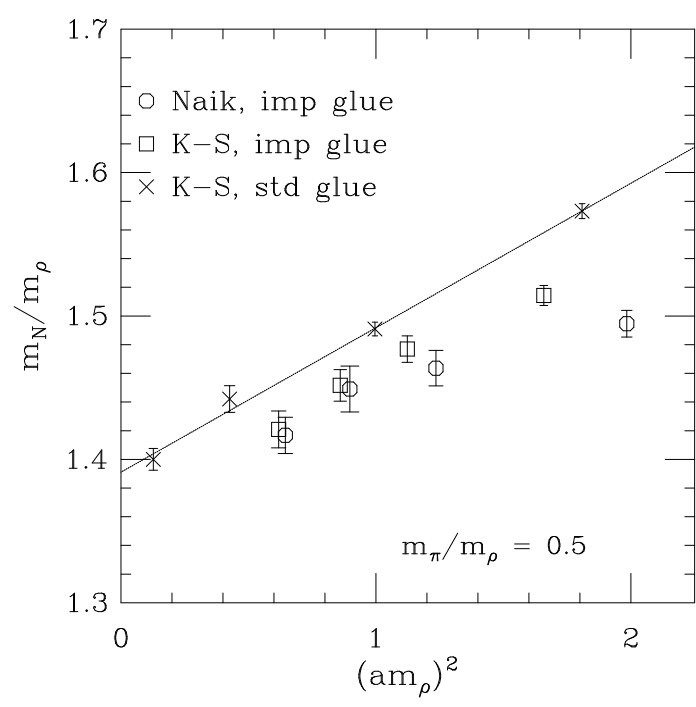

Figure 4. Scaling plot of nucleon to $\rho$ mass ratio for quark mass such that $m_{\pi} / m_{\rho}=0.5$.

so there is a difference between the staggered and improved quark actions even for $a m_{\rho} \leq 1$.

We find a modest improvement to flavor symmetry restoration and the nucleon to rho mass ratio by using an improved gluon action with either Kogut-Susskind or Naik quarks when $\operatorname{am}_{\rho}\left(m_{q} \approx\right.$ $0) \approx 1.2$. For smaller lattice spacing, it is possible that adding additional terms as detailed by Luo [5] will lead to an action with errors of order $a^{4}$. More work is required to do precision physics with these actions or more refined ones.

This work was supported by the DOE and the NSF. Computations were done at Indiana University, PSC, SCRI and SDSC.

\section{REFERENCES}

1. For a review of improvement, see G. P. Lepage, Nucl. Phys. (Proc. Suppl.) 47 (1996) 3.

2. B. Sheikholeslami and R. Wohlert, Nucl. Phys. B259 (1985) 572.

3. C. Bernard et al., Nucl. Phys. (Proc. Suppl.) 53 (1997) 212.

4. S. Naik, Nucl. Phys. B316 (1989) 238.

5. Y. Luo, Phys. Rev. D55 (1997) 353; heplat/9702013. 
6. T. Blum et al., Phys. Rev. D55 (1997) 1133.

7. S. Collins et al., Nucl. Phys. (Proc. Suppl.) 47 (1996) 378. 